\title{
Multi-physics Field Analysis of Nuclear Power Valve based on MDO Model
}

\author{
Dai Ye*, Jiang Jin-gang and Cheng Tian-yu \\ School of Mechanical \& Power Engineering, Harbin University of Science and \\ Technology, Heilongjiang Harbin 150080, China \\ *daiye312@163.com
}

\begin{abstract}
In order to construct the characteristics evaluation system of nuclear power valves, multiphysics analysis (including structural static analysis, thermal-mechanical coupling analysis, flow field analysis and Seismic analysis) are completed on a typical nuclear power valve based on finite element analysis (FEA). Binding the load standard of ASEM, each performance index of the nuclear power valve is tested and forecasted in order to verify the safety performance. Meanwhile, in view of the demands in multidisciplinary design optimization (MDO) of nuclear power valve products, the MDO model for valves is established, which integrates multi-characteristic simulation and multidisciplinary design of nuclear power valves together. The validity of the analysis method above is verified by the valve strength and sealing tests, which is of certain guiding significance for other class of nuclear power valves.
\end{abstract}

Keywords: multi-physics analysis; multidisciplinary design; nuclear power valve

\section{Introduction}

In the numerical calculation and analysis of nuclear power valve design, valve body is the main stressed components, which often embody in the irregular and relatively complex geometry, the complex work environment, and the impact damage of valve cavity under the working medium in different flow velocity, temperature, and chemical properties, hence, the analysis work of nuclear power valve is very complex [1-4]. In conventional nuclear power valve design, CAE experts in different fields are indispensable to participate in the multiphysics field analysis. In fact, the analysis contents and evaluation methods for similar nuclear power valve products are basically same, then setting up the analysis system for a certain nuclear power valve also has guiding significance to the similar value products [5-9]. At the same time, the establishment of the analysis system for nuclear power valve provides the navigation process for the MDO integrated system, and the analysis process can be converted to the analysis templates that can be used in the process of analysis reuse [10].

\section{Establishment of MDO Model for Nuclear Power Valves}

In order to comprehensively analyze the specific impact of the nuclear power valve under various influence factors, multi-physics field analysis of the whole valve, including structural static analysis, thermal-mechanical coupling analysis, flow field analysis, Seismic analysis, can be completed to predict the valve performance under different work conditions [11]. Figure 1 illustrates the MDO model for nuclear power valves. MDO 
in valve design in this paper is divided into four parts: mechanical analysis, fluid mechanics analysis, thermal-mechanical coupled analysis and seismic analysis, which are totally based on the boundary and constrain condition of the actual working conditions so as to conform to the engineering practice. And the mixed model of simulation and optimization which contains structural features and boundary conditions is also founded. This hierarchical model can be divided into three levels: system optimization model, subsystem optimization model and subsystem analysis model. The three levels not only ensure the total optimization property of the entire product, but also give consideration to the independence of each subsystem so as to realize the collaboration design easily between different areas.

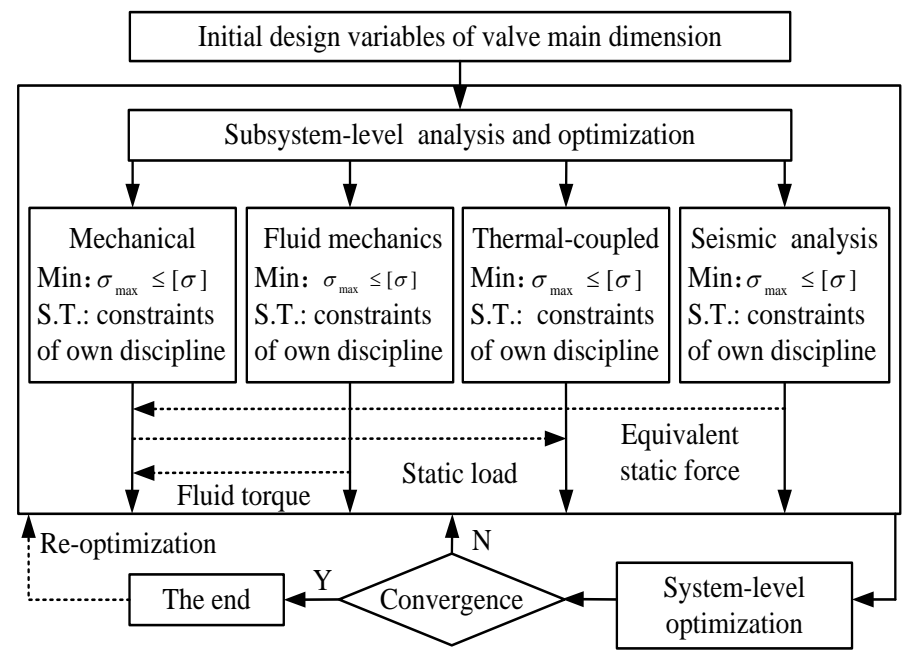

Figure 1. MDO model for valves

\section{Multi-physics Field Analysis of Nuclear Power Valve}

Because the valve structure is complicated, conventional analytical method can only calculate the mean stress of the various parts through the empirical formula or a rough formula, so it can not meet the actual needs of engineering. Hence, the finite element analysis has been used for the analysis by ANSYS, in which the coupling analyses under working condition are completed. Due to the structure design of gate valve is the most representative, so the supercritical electric gate valve (DN230-CL2680) is selected as an example to study. The main performance parameters of the electric gate valve as shown in Table 1.

Table 1. Main performance parameter of gate valve DN230-CL2680

\begin{tabular}{cccccccc}
\hline $\begin{array}{c}\text { Pressure } \\
\text { Class }\end{array}$ & $\begin{array}{c}\text { Design } \\
\text { Pressure } \\
/ \mathrm{MPa}\end{array}$ & $\begin{array}{c}\text { Design } \\
\text { Temperature } \\
{ }^{\circ} \mathrm{C}\end{array}$ & $\begin{array}{c}\text { Working } \\
\text { Pressure } \\
/ \mathrm{MPa}\end{array}$ & $\begin{array}{c}\text { Working } \\
\text { Temperature } \\
/{ }^{\circ} \mathrm{C}\end{array}$ & $\begin{array}{c}\text { safety } \\
\text { level }\end{array}$ & $\begin{array}{c}\text { anti- } \\
\text { seismic } \\
\text { leve }\end{array}$ & $\begin{array}{c}\text { Connection } \\
\text { type }\end{array}$ \\
\hline $\begin{array}{c}\text { Class } \\
2680\end{array}$ & 30.1 & 441 & 27.5 & 365 & First & First & BW \\
\hline
\end{tabular}

\subsection{Structural analysis of valve body}

According to the analysis of different working conditions, the most complex conditions of the valve body is when the valve is in the fully closed position. In this condition, the load 
suffered by valve can be obtained as : the pressure in pressure cavity of valve body is $27.5 \mathrm{MPa}$, and the temperature is $365^{\circ} \mathrm{C}$. In addition, all analyses will use identical boundary conditions as to be comparable.

According to the parameters provided by the ASEM (American Society for Engineering Management), the load combination coefficient $k=1$, design stress intensity $S_{m}=145 \mathrm{MPa}$, $S_{a}=500 \mathrm{MPa}$ as the switch cycle life is 1000 times. After analysis, valve body stress distribution diagram can be obtained, from which it could predicted that the maximum primary stress appears at the intersection of the valve cavity and the import tube (and export tube) as shown in Figure 2, this is in line with the actual situation. Therefore, the following structural strength checking is mainly around these areas.

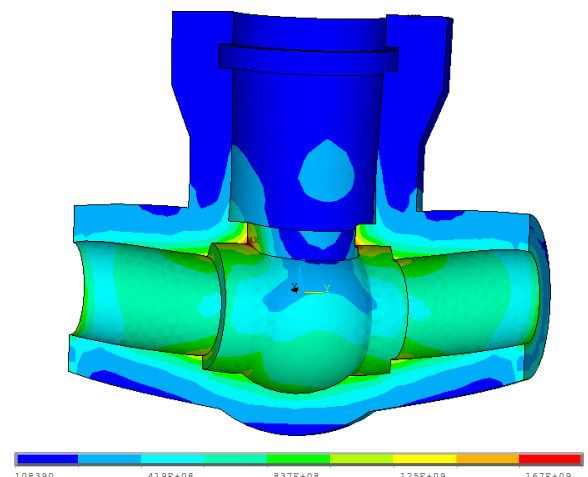

Figure 2. Valve body stress distribution

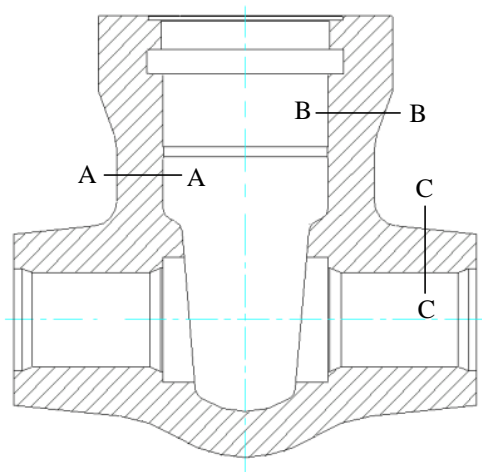

Figure 3. Computation section position

Taking the section A-A as the calculation section as shown in Figure 3, the cross section stress is only caused by the design internal pressure of $30.1 \mathrm{MPa}$, and does not include the discontinuity of stress concentration, namely, the stress is primary stress on this cross section as shown in Figure 4.

Taking the mean of the absolute value (membrane stress), $\bar{\sigma}_{1}=86.63 \mathrm{MPa}$, $\overline{\sigma_{2}}=42.21 \mathrm{MPa}, \overline{\sigma_{3}}=16.82 \mathrm{MPa}$. Hence, the total membrane stress of the primary stress meets the strength requirement, i.e., $\mathrm{P}_{\mathrm{m}}=\max \left\{\overline{\sigma_{1}}, \overline{\sigma_{2}}, \overline{\sigma_{3}}\right\}<k S_{\mathrm{m}}=145 \mathrm{MPa}$. Taking the section B-B as the calculation section, the cross section is suffered by the design internal pressure of $30.1 \mathrm{MPa}$ and the local discontinuous structures (the radius change), and does not include the stress concentration as shown in Figure 5.

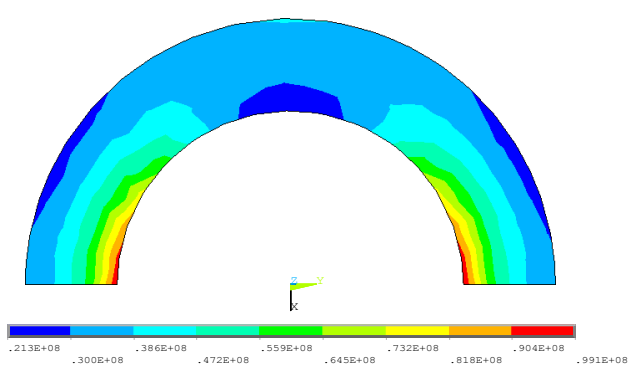

Figure 4. Stress distribution on A-A Section

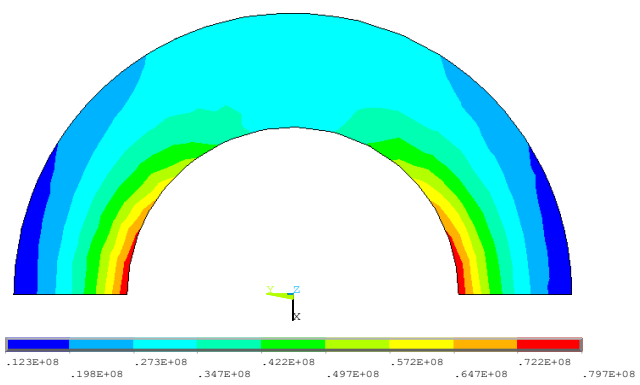

Figure 5. Stress distribution on B-B Section 
The bending stress is only generated at end socket, hence, the section C-C is selected as the calculation section, from which it can be known that $\sigma_{1 \max }=68.82 \mathrm{Mpa}, \sigma_{2 \max }=46.32 \mathrm{Mpa}$, $\sigma_{3 \max }=29.75 \mathrm{Mpa}$, hence, the local membrane stress of the primary stress added to the bending stress meets the strength requirement, namely, $P_{L}=\max \left\{\overline{\sigma_{x}}, \overline{\sigma_{y}}, \overline{\sigma_{z}}, \overline{\tau_{\mathrm{xy}}}, \overline{\tau_{\mathrm{y} z}}, \overline{\tau_{z x}}\right\}<1.5 k S_{\mathrm{m}}=217.5 \mathrm{MPa}$.

\subsection{Thermal-structure coupling analysis of valve body}

There are kinds of thermal-structure coupling analysis in ANSYS, sequential coupling method and direct coupling method. The sequential coupling method is adopted in this paper, the principle of which is to take the node temperature obtained by thermal analysis as body force load that is applied in the stress analysis to achieve coupling process, and then the thermal analysis results are loaded directly into the stress analysis through thermal-stress conversion elements, and the thermal analysis can be directly converted to stress analysis in ANSYS. In order to reduce the solving difficulty, the outer surface of the valve body in the analysis can be regarded as adiabatic boundaries, and the inner surface is convective heat transfer boundary, which is the third boundary condition. The finite element analysis model is shown in Figure 6, the symmetric cutting plane is suffered to the adiabatic boundary condition.

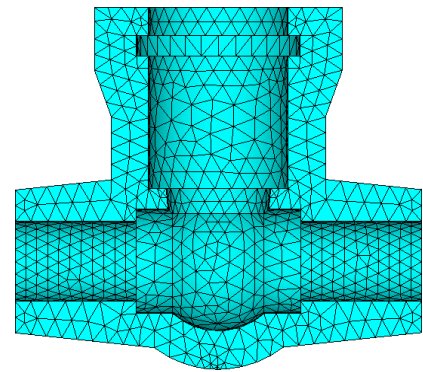
Figure 6. Finite element model of valve body

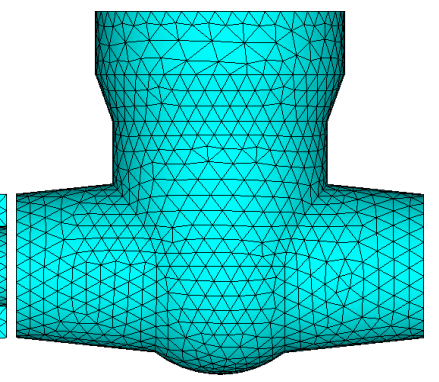

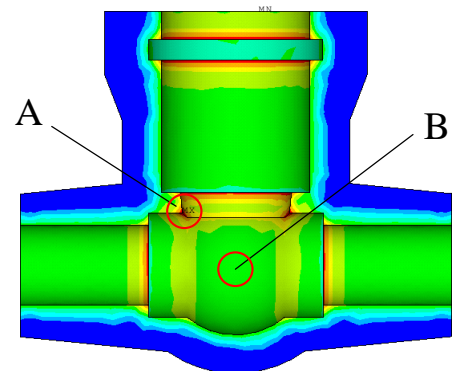

Figure 7. Temperature vector diagram

As shown in Figure 7, it is the temperature vector diagram of the valve body after starting 1 min, from which it can be known that the regional isotherm basic parallel to the valve wall, which indicates that there are mainly radial temperature difference in each region. Here a contrastive analysis is completed at the cutting-edge point $\mathrm{A}$ and the point $\mathrm{B}$ near inner valve cavity as shown in Figure 9. 


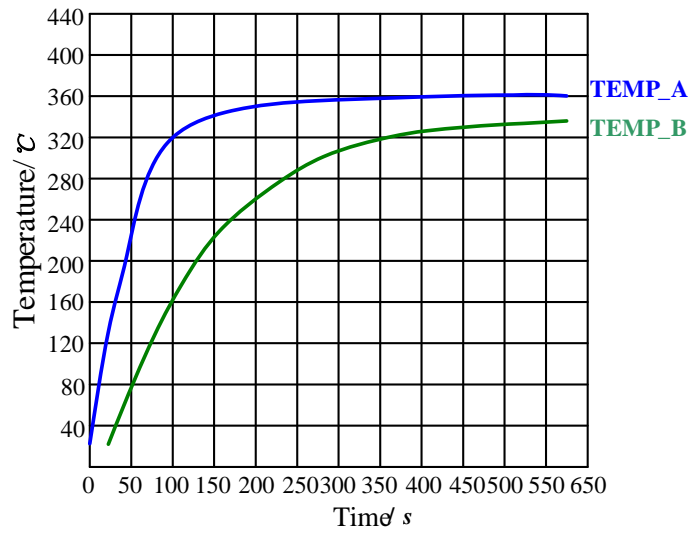

Figure 8. A and B temperature curve

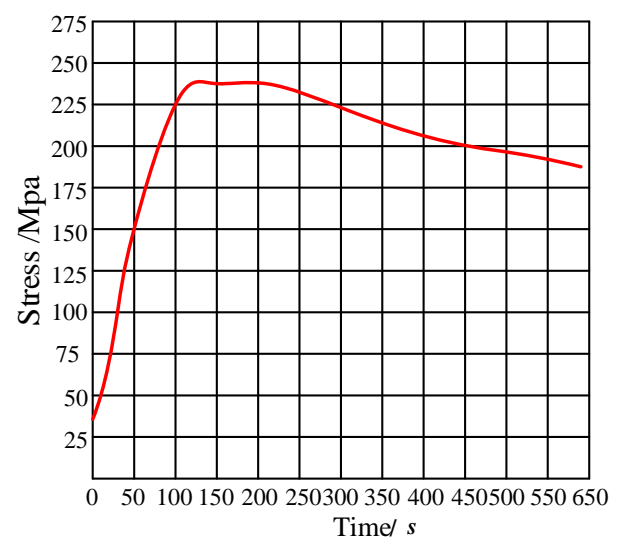

Figure 9. Stress variation curve of point $A$

The curves of temperature change over time at point $\mathrm{A}$ and point $\mathrm{B}$ are shown diagrammatically in Figure 8. In the process of starting, the equivalent heat transfer between attachment area near point $\mathrm{A}$ and high temperature steam, so the temperature rise is faster at point A within the first 100 seconds, the temperature reached $375{ }^{\circ} \mathrm{C}$ at the 124 th second; Due to the material properties of heat retardance, temperature rising rate is relatively slower at point $\mathrm{B}$, the temperature change becomes placid after $410 \mathrm{~s}$. As shown in Figure 9, it is the stress changing curve of von Mises $\sigma$ change over time at point A. It can be seen from the diagram that the total stress (the sum of thermal stress and pressure of the steam) reaches the peak value of $264 \mathrm{MPa}$ around 126s, this process lasts only about 80 seconds.

\subsection{Flow field analysis of the valve cavity}

The process discrete analysis method is adopted to analyze fluid characteristics in the opening and closing process of the valve. On a certain relative position, the fluid in the valve cavity flow steady through the valve plate, at this time, the flow belongs to steady fluid. The whole opening and closing process of valve is divided in to five different positions by using the valve opening interval of $20 \%$ (i.e., the opening of $20 \%, 40 \%, 60 \%, 40 \%, 60 \%$ and $100 \%)$.

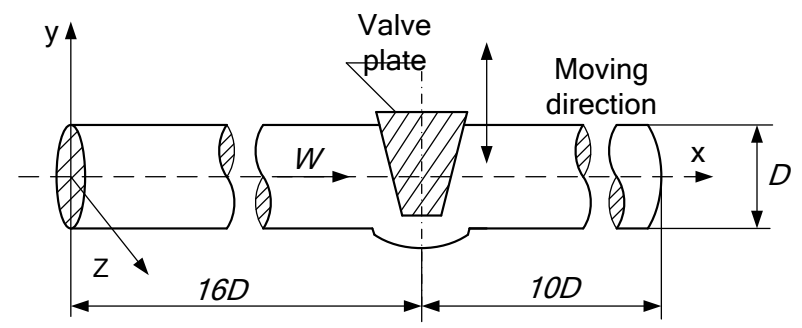

Figure 10. Flow field analysis of valve cavity

In the process of establishing the fluid finite element model, the model boundary should be far away from the large gradient areas in the flow field, so the fluid calculation area near import and export position should be extended appropriately. According to the empirical equation $L_{e} / D=4.4\left(R e_{D}\right)^{1 / 6}$ given by Muson ${ }^{[12]}$, the length of import and export are $16 D$ and $10 D$ respectively, the analysis model is shown in Figure 10. The finite volume method and the standard $k-\varepsilon$ turbulence are used to carry out the flow field analysis. 

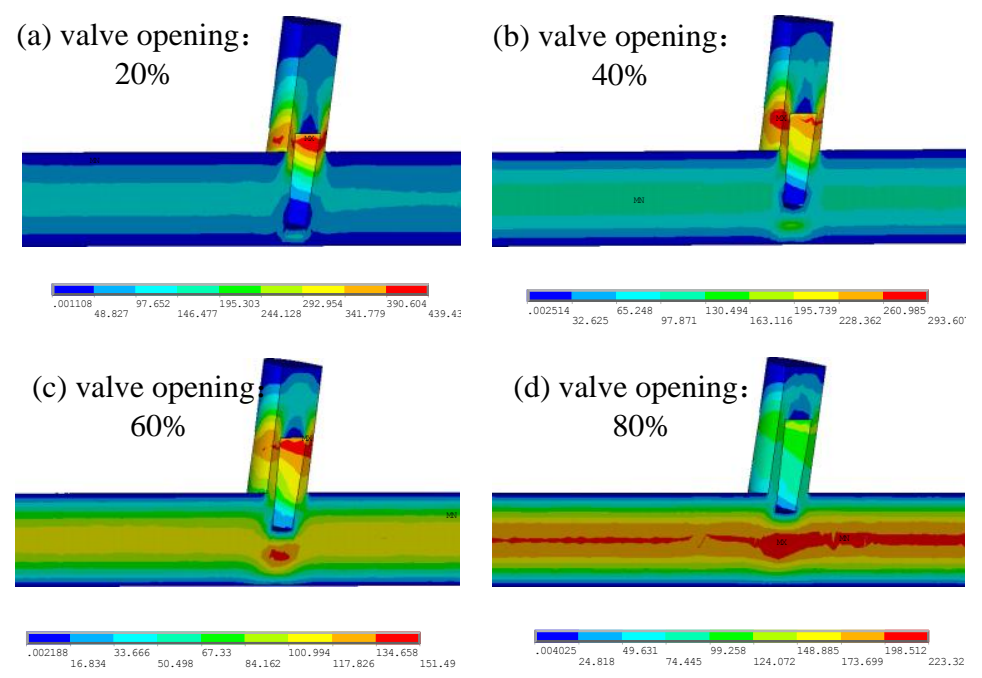

\section{Figure 11. Flow velocity distribution with different opening positions}

Figure 11 shows the pressure and velocity distribution under different opening in the valve cavity flow field, the analysis results show that the static pressure and fluid velocity in the valve cavity have a large change in the opening and closing process of the gate valve. According to different opening position, the flow characteristic in the valve cavity can be obtained as shown in Table 2. According to the fluid mechanics, the fluid resistance loss can be expressed by the fluid pressure drop $\Delta P$, for the turbulent flow regime, $\Delta P=\zeta v^{2} \rho / 2$, then $\zeta=2 \Delta P / v^{2} \rho$, where $\zeta$ is the flow resistance coefficient of the valve, $\rho$ is the fluid density, $\boldsymbol{V}$ is the mean fluid velocity in the valve cavity.

Table 2. Flow performance of electric gate valves DN230CL2680

\begin{tabular}{cccc}
\hline $\begin{array}{c}\text { Valve opening } \\
\%\end{array}$ & $\begin{array}{c}\text { Export flow velocity } \\
\mathrm{m} / \mathrm{s}\end{array}$ & $\begin{array}{c}\text { Flow ratio } \\
\%\end{array}$ & $\begin{array}{c}\text { Flow resistance } \\
\text { coefficient }\end{array}$ \\
\hline 0 & 0 & 0 & -- \\
20 & 97 & 46.41 & 16.13 \\
40 & 119 & 56.93 & 1.84 \\
60 & 152 & 72.72 & 0.65 \\
80 & 193 & 92.34 & 0.26 \\
100 & 209 & 100 & 0.08 \\
\hline
\end{tabular}

\subsection{Seismic performance analysis of the valve assembly}

In the seismic analysis of valve, if the lowest natural vibration frequency of the valve is more than $33 \mathrm{~Hz}$, then using the equivalent static method should be used in the stress calculation. On the premise of ensuring the calculation precision, the valve clack, valve rod, and guide plate has made the simplify processing, the mass of these parts are added approximately to the main parts close to their center of mass, the total mass of $421 \mathrm{~kg}$ of the valve stays the same, the finite element analysis model is shown in Figure 12. 


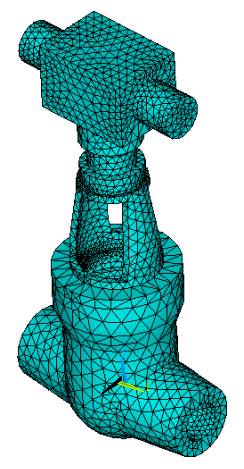

Figure 12. Dynamic analysis model

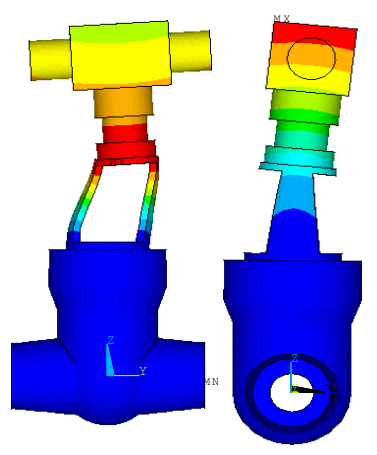

Figure 13. First four order modes of the valve assembly

It can be known from the dynamic analysis results as shown in Figure 13 that the first order modal value is more than $33 \mathrm{~Hz}$, so the equivalent static method should be adopted to make the earthquake-resistance analysis, the purpose of which is to check if the nuclear grade valve during or after the earthquake can guarantee the integrity of the structure. On the safe shutdown earthquake (SSE), Valves shall meet the NB-3500 standard requirement of ASME III volume. The earthquake acceleration values (excluding the gravity acceleration) in the three direction of $\mathrm{X}, \mathrm{Y}, \mathrm{Z}$ are respectively $3 \mathrm{~g}$; on the operating basis earthquake (OSE), the earthquake acceleration values (excluding the gravity acceleration) in the three direction of $\mathrm{X}, \mathrm{Y}, \mathrm{Z}$ are respectively $2 \mathrm{~g}$. In order to make the valve safer, according to the design pressure load and the design temperature under the allowable stress strength for analysis and evaluation. Table 3 lists the stress calculation results of the key parts. The analysis results show that the stress values under earthquake conditions are less than the corresponding limits, and there is a certain safety margin, so this gate valve meets the seismic requirements.

Table 3. Seismic resistance analysis

\begin{tabular}{|c|c|c|c|}
\hline Key parts & $\begin{array}{c}\text { Stress value } \\
(\mathrm{MPa})\end{array}$ & $\begin{array}{c}\text { Allowable stress intensity } \\
(\mathrm{MPa})\end{array}$ & $\begin{array}{l}\text { Seismic } \\
\text { capacity }\end{array}$ \\
\hline $\begin{array}{l}\text { Transition area of } \\
\text { valve and pipe }\end{array}$ & $\begin{array}{c}P_{\mathrm{L}}=90.86 \\
P_{\mathrm{L}}+P_{\mathrm{b}}=156.73\end{array}$ & $\begin{array}{l}1.5 k S_{\mathrm{m}}=1.5 \times 145=217.5 \\
1.5 k S_{\mathrm{m}}=1.5 \times 145=217.5\end{array}$ & Safety \\
\hline $\begin{array}{l}\text { Stress concentration } \\
\text { area of the valve body }\end{array}$ & $P_{\mathrm{L}}+P_{\mathrm{b}}+Q=171.61$ & $3 k S_{\mathrm{m}}=3 \times 145=435$ & Safety \\
\hline $\begin{array}{l}\text { Transition area of } \\
\text { valve deck and body }\end{array}$ & $\begin{array}{c}P_{\mathrm{L}}=37.24 \\
P_{\mathrm{L}}+P_{\mathrm{b}}=57.38\end{array}$ & $\begin{array}{l}1.5 k S_{m}^{\prime}=1.5 \times 94.5=141.75 \\
1.5 k S_{m}^{\prime}=1.5 \times 94.5=141.75\end{array}$ & Safety \\
\hline $\begin{array}{l}\text { Stress concentration } \\
\text { area of the valve deck }\end{array}$ & $P_{\mathrm{L}}+P_{\mathrm{b}}+Q=47.17$ & $3 k S_{m}^{\prime}=3 \times 94.5=103.95$ & Safety \\
\hline $\begin{array}{l}\text { Stress concentration } \\
\text { area of the holder }\end{array}$ & $P_{\mathrm{L}}+P_{\mathrm{b}}+Q=76.36$ & $3 \mathrm{kS}_{\mathrm{m}}^{\prime \prime}=3 \times 114.5=377.85$ & Safety \\
\hline
\end{tabular}

Where : $k$ is the load combination coefficient, here $k=1 ; S_{\mathrm{m}}, S_{\mathrm{m}}^{\prime}$ and $S_{\mathrm{m}}^{\prime \prime}$ are the design stress intensity values respectively of corresponding parts material in ASEM.

\section{Strength and Sealing Test of the Valve}

Through the analysis and evaluation system of the more multi-physical field above, various performances of the valve under the working conditions have been predicted. In order 
to verify the structural strength and sealing of the valve body, the hydrostatic test is used as shown in Figure 14.

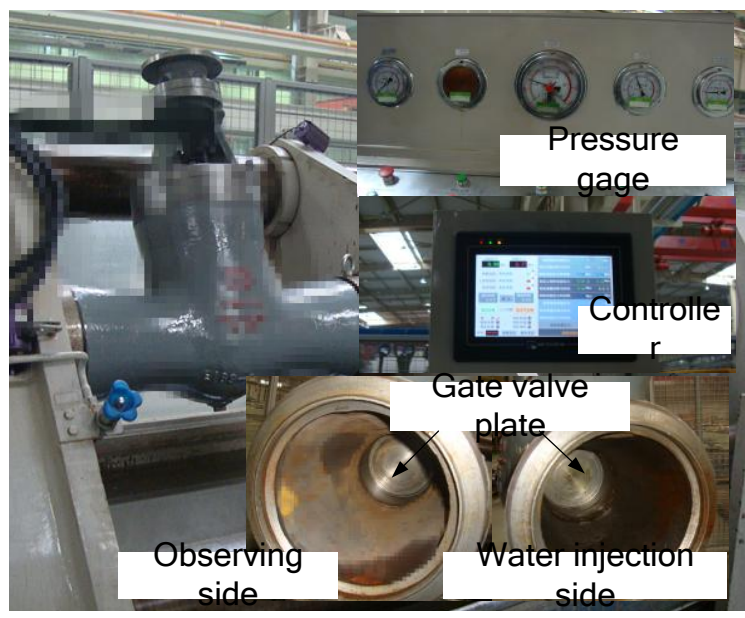

\section{Figure 14. Hydraulic pressure testing for valve}

The design pressure of the gate valve is $30.1 \mathrm{MPa}$. The hydraulic pressure should be at least 1.5 times the maximum working pressure in the strength test, and be at least 1.1 times in the sealing test, so the experimental pressure is $46 \mathrm{MPa}$ and $34 \mathrm{MPa}$, respectively. The hydraulic pressure should be loaded gradually from $30 \mathrm{MPa}$ to $46 \mathrm{MPa}$, holding time for $5 \mathrm{~min}$. Test results: in the pressurized process, there is no medium leakage phenomenon and welding crack on the body. In the whole sealing test, there is also no medium leakage phenomenon. Hence, the test results show that the nuclear power valve body meets the work requirements in terms of the strength and sealing performance, and which confirms the correctness of the method and the calculation process.

\section{Conclusion}

To construct the characteristics evaluation system of nuclear power valves, multi-physics analyses are completed on the supercritical electric gate valve (DN230-CL2680).

(1) In view of the demands in multidisciplinary design optimization (MDO) of valve products, the MDO model for valves is constructed, which takes account of mechanical analysis, fluid mechanics analysis, thermal-mechanical coupled analysis and seismic analysis.

(2) On this basis of MDO model, the multi-physics analyses of the electric gate valve of DN230-CL2680 are carried out, which can predict a variety of working characteristics by FEA and perfect the technical standard and the norm of nuclear power valve design. The simulation of the valve can effectively improve the accuracy and efficiency of designing the valve products.

(3) The validity of the analysis method above is verified by the valve strength and sealing tests, which results show that the nuclear power valve body meets the work requirements in terms of the strength and sealing performance that confirms the correctness of the method and the calculation process. The method is of certain guiding significance for other class of valves. 


\section{Acknowledgements}

This research was supported by the National Natural Science Foundation of China (Grant No. $51105120)$.

\section{References}

[1] S. Bing and J. Ye, "Study on the Stem Heat Expansion and Its Influence of the Reheat Stop Valve in 600 MW Steam Turbine Unit", Journal of Power Engineering, vol. 28, no. 4, (2008), pp. 573-578, (in Chinese).

[2] X. G. Song and L. C. Park, "Three-Dimensional CFD Analysis of A Spring-Loaded Pressure Safety Valve from Penning to Re-Closure", The ASME 2010 Pressure vessels \& Piping Division Conference, vol. 3, (2010), pp. 18-22.

[3] D. Ye, L. Yinan and G. D. Todorov, "Structural optimization of high pressure bypass-valve body for 600MW supercritical units", Journal of Harbin Institute of Technology (New Series), vol. 17, no. 1, (2010), pp. 168170.

[4] S. X. Guan, C. Lei and P. Y. Chu, "Three-dimensional CFD analysis of a spring-loaded pressure safety valve from opening to re-closure", American Society of Mechanical Engineers, vol. 5, (2010), pp. 295-303.

[5] X. G. Song and L. Wang, "Fluid and structural analysis of a large diameter butterfly valve", Journal of Advanced Manufacturing Systems, vol. 8, no. 1, (2009), pp. 81-88.

[6] Y. L. Perry, "Using steady flow force for unstable valve design: modeling and experiments", Department of Mechanical Engineering, vol. 27, (2005), pp. 39-42.

[7] X. M. Yu and L. W. Zhong, "Numerical Simulation of Vibration Performance For Nuclear Island Radioactive Boron Acid Water Control Valve", 2010 International Conference on Mechanic Automation and Control Engineering, (2010), pp. 3986-3989.

[8] Salloom, Y. Maher and Samad, "Finite element modeling and simulation of proposed design magnetorheological valve", International Journal of Advanced Manufacturing Technology, vol. 54, no. 8, (2011), pp. 421-429.

[9] R. Amirante, G. D. Vescovo and A. L. Ippolis, "A flow forces analysis of an open center hydraulic directional control valve sliding spool”, Energy Convers Manage, vol. 47, (2006), pp. 114 -131.

[10] D. Ye, L. Yinan and H. Xibin, "Design and implementation of MDO integrated system for valve products", Advanced Materials Research, vol. 308, (2011), pp. 1273-1276.

[11] G. S. Xue, S. G. Kim and L. Wang, "Transient flow analysis of spring loaded pressure safety valve", American Society of Mechanical Engineers, vol. 7, (2010), pp. 253-258.

[12] B. R. Munson, D. F. Young and T. H. Okiishi, "Fundamentals of Fluid Mechanics", John Wiley Inc, (2002). 
International Journal of Control and Automation Vol.7, No.4 (2014) 